\title{
The fcc/hcp Relative Phase Stability in the Fe-Mn-Co System: Martensitic Transformation Temperatures, Assessment of Gibbs Energies and Thermodynamic Calculation of $\mathbf{T}_{0}$ Lines
}

\author{
A. Baruj, A. Fernández Guillermet* and M. Sade \\ Centro Atómico Bariloche, CNEA, 8400 Bariloche, Argentina \\ * CONICET, Argentina
}

\begin{abstract}
In this paper the results of a systematic study of the fcc/hcp relative phase stability in the Fe-Mn-Co system are presented, which is based on new, extensive measurements of the martensitic transformation temperatures. The whole range of compositions where the transformation occurs has now been covered. A thermodynamic analysis of the experimental information based on Gibbs energy modelling of the Fe-Mn-Co, Fe-Mn and Fe-Co systems is performed. The $\mathrm{T}_{0}$ temperatures for the Fe-Mn-Co system are established. It is shown how ternary information can be utilized to assess the Gibbs energy function of the hcp phase, which is not stable in the Fe-rich region of the binary Fe-Co system.
\end{abstract}

\section{INTRODUCTION}

Considerable effort has been devoted over the years to develop databases with assessed thermodynamic information on metals, their binary and higher-order alloys. Such an activity has been stimulated by the recognition that thermodynamics offers a key route to understanding the macroscopic behaviour of real systems, and provides an additional basis for designing new materials. Establishing the thermodynamics of a multicomponent alloy system is a demanding task, which requires relatively large amounts of experimental information. However, such information might not be available. In fact, the data that are involved in the evaluation of the Gibbs energy function of stable phases are frequently scarce, unreliable or completely lacking. A particularly difficult case is encountered when the phase that is the target of the assessment is not stable in the ranges of chemical composition, temperature or pressure that can be reached by the current experimental techniques. When focussing on the fcc/hcp relative stabilities in Fe-Mn-Co alloys one faces both kinds of problems. Firstly, there is a martensitic transformation (MT) between these phases, but the experimental information about it is scarce [1-3]. Secondly, the fact that the hep phase in Fe-rich Fe-Co alloys is not stable under ordinary conditions makes it difficult to determine accurately the Gibbs energy function of this phase [4]. In a previous work [5] we presented information about the Fe-Mn-Co system covering the range of $\mathrm{Mn}$ contents in which the MT takes place, but for low Co contents, only. We also explored the possibility of using ternary information in refining the Gibbs energy function for the hcp phase in the Fe-Co system [5]. An open question is, however, how the iso- $A_{s}$ and iso-M lines for the fcc/hcp MTs in the Fe-Mn-Co system behave at high Co contents, and what is the result of using such ternary information in assessing the $\mathrm{fcc} / \mathrm{hcp}$ stability in Fe-rich region of the Fe-Co system. Another point of interest is the presence of a third martensitic phase in this alloys: a bcc ( $\alpha$ ) phase. In Fe-Mn alloys this phase forms martensitically only in alloys with less than about $16 \mathrm{wt} \% \mathrm{Mn}[6,7]$. However, it is not known how this limit changes with the addition of $\mathrm{Co}$, and how the presence of the $\alpha$ phase does affect the temperatures for the start of the fcc/hcp MT. The purpose of this work is to extend our previous study of the fcc/hcp MT in Fe-Mn-Co alloys [5] to regions of low Mn contents ( $<16 \mathrm{wt} . \%)$, as well as to high Co contents ( $>8 \mathrm{wt} . \%)$. With this new information a full thermodynamic analysis will also be performed. 


\section{EXPERIMENTAL TECHNIQUES}

The preparation of Fe-Mn-Co alloys was described previously [5]. Briefly, pure elements were arc-melted several times under an Ar atmosphere. The resulting alloys were encapsulated in quaitz under an Ar atmosphere, kept 48 hours at $1273 \mathrm{~K}$ and quenched in water at room temperature. Fe-Mn-Co samples for dilatometric and electrical resistivity measurements were spark cut, encapsulated in quartz under $\mathrm{Ar}$, annealed 1 hour at $1273 \mathrm{~K}$ and finally quenched in water. The MT temperatures were determined either by dilatometry or by electrical resistivity measurements, with a Chromel-Alumel thermocouple welded on the sample. Both equipments are home-made and work in the range from $190 \mathrm{~K}$ to $680 \mathrm{~K}$. In order to determine if the bcc $(\alpha)$ martensitic phase was present in the alloys, an indirect experimental method which uses an Adamel-Lhomargy LK-02 dilatometer was applied. The method has been explained in detail in ref. [8]. Here we give only the main points. The MT from the fcc phase to the bcc phase occurs at a higher temperature than the hcp/fcc MT and has an opposite volume change. The existence and formation of the $\alpha$ phase was investigated after the measurement of the fcc/hcp MT temperatures by performing an extra heating-cooling cycle between room temperature and $1273 \mathrm{~K}$ in the Adamel dilatometer. Small pieces from the samples were spark cut for analysing its chemical composition. The analysis was carried out by using the wave dispersive energy (WDS) technique in a Phillips scanning electron microscope and the pure metals as standards. In this paper all the compositions are given in weight percentage and all the temperatures are in K.

\section{TRENDS IN $M_{\mathrm{s}}$ AND A $\mathrm{A}_{\mathrm{s}}$ TEMPERATURES}

The MTs determined as described in Section 2 are represented in Fig. 1 In this figure each data point represents the composition of an alloy, and the number close to it is the MT temperature measured dilatometrically. The $\alpha$ symbol close to a point indicates that the martensitic bcc phase was determined to be present in the sample. The samples with $\mathrm{Mn}$ contents up to $12 \%$ and the alloy with $13.9 \% \mathrm{Mn}-14.1 \% \mathrm{Co}$ do not show the $\mathrm{fcc} / \mathrm{hcp}$ MT. However, in these alloys the fcc/bcc MT was detected. The alloys with $13.2 \% \mathrm{Mn}-4.8$ $\% \mathrm{Co}$ and $14.3 \% \mathrm{Mn}-9.0 \% \mathrm{Co}$ show a slight hcp/fcc MT. It was not possible to measure the $\mathbf{M}_{\mathrm{s}}$ temperature on cooling, but a subsequent heating revealed the occurrence of the hcp/fcc MT. It was found that the $\alpha$ phase was also present in these alloys in the temperature range where the fcc/hcp and hcp/fcc MTs occur. The same was observed in alloys with $14.7 \% \mathrm{Mn}-3.1$ $\% \mathrm{Co}, 15.5 \% \mathrm{Mn}-12.9 \% \mathrm{Co}, 17.6 \% \mathrm{Mn}-11.8$ $\% \mathrm{Co}$ and $18.3 \% \mathrm{Mn}-16.2 \% \mathrm{Co}$. In this group the $\mathrm{fcc} / \mathrm{hcp}$ and $\mathrm{hcp} / \mathrm{fcc}$ MTs were well detected, but it is quite possible that the presence of the $\alpha$ phase might have influenced the observed fcc/hcp MT temperatures. We return to this point in Section 5. These alloys have been used to determine the boundaries of the region where the $\alpha$ phase can form. In the alloys located to the right of this boundary in Fig. 1, the fcc/hcp and hcp/fcc MTs occur in the absence of the $\alpha$ phase. The dashed

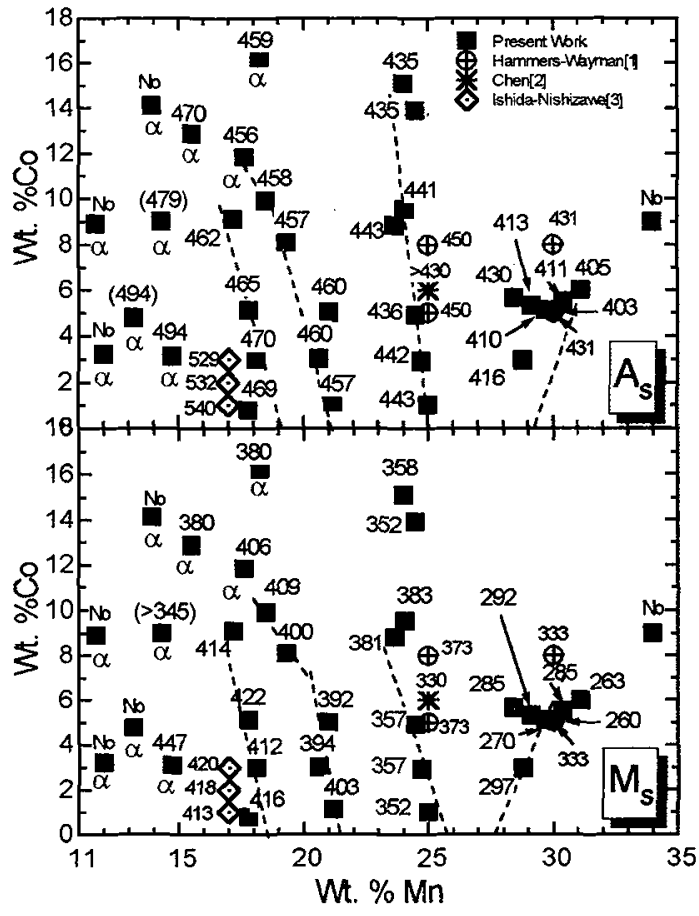

Fig. 1: $A_{s}$ and $M_{s}$ temperatures for Fe-Mn-Co alloys measured. Data from refs. [1-3] are given for comparison. Each point represents an alloy and the number close to it is the measured transformation temperature in $K$. The dashed lines are guides to the eye. 
lines in Fig. 1 connect points with the same values of $M_{s}$ and $A_{s}$. In the alloys with $\mathrm{Mn}$ contents between about $18 \%$ and $25 \%$ the iso- $A_{s}$ and iso- $M_{s}$ lines tend to deviate from the vertical towards the Co axis. For Co contents lower than about $9-10 \%$ this tendence is slight, i.e., low Co additions have a small effect upon the MT temperatures of the Fe-Mn alloys. At higher Co contents the deviation is stronger, indicating a stabilization of the fcc phase with respect to the hcp phase. At higher $\mathrm{Mn}$ contents, i.e. above $25 \% \mathrm{Mn}$, the slope of the iso- $A_{s}$ and iso- $M_{s}$ lines indicate a relative stabilization of the hcp phase. Such a qualitative change in behaviour of the iso- $\mathrm{M}_{\mathrm{s}}$ and iso- $\mathrm{A}_{\mathrm{s}}$ lines is essentially due to the existence of an antiferromagnetic ordering reaction in the fcc phase, the effects of which have been discussed in our previous papers [5-7]. Here we only comment on the composition dependence of the Néel temperature of the fcc phase $\left(T_{\mathbb{N}}^{\mathrm{fcc}}\right)$ in the Fe-Mn-Co alloys. $\mathrm{T}_{\mathrm{N}}^{\mathrm{fcc}}$ increases with the $\mathrm{Mn}$ content $[6,7]$, but is decreased with increasing Co additions [9]. The latter effect destabilizes fcc and thus promotes the $\mathrm{fcc} / \mathrm{hcp} \mathrm{MT}$. The region where the qualitative change in behaviour of the iso- $\mathrm{M}_{\mathrm{s}}$ and iso- $\mathrm{A}_{\mathrm{s}}$ lines occurs is located around $25 \% \mathrm{Mn}$. There $\mathrm{T}_{\mathrm{N}}^{\mathrm{fcc}}$ is close to or falls between $A_{s}$ and $M_{s}$. Since the antiferromagnetic ordering reaction plays a key role in determining the fcc/hcp relative stability in Fe-Mn [5-7], and Fe-Mn-X [5] alloys, new magnetic measurements are currently carried out by us in order to establish in detail the composition dependence of $\mathrm{T}_{\mathrm{N}}^{\mathrm{fcc}}$ in the Fe-Mn-Co system as well as in other Fe-Mn-X systems.

\section{THERMODYNAMIC ANALYSIS}

\subsection{Gibbs Energy Modelling}

The $\mathrm{G}_{\mathrm{m}}$ function of the fcc or hcp phase in the Fe-Mn-Co system is described by the following expression:

$$
\begin{aligned}
\mathrm{G}_{\mathrm{m}}^{\phi} & =\mathrm{x}_{\mathrm{Co}}{ }^{0} \mathrm{G}_{\mathrm{Co}}^{\phi}+\mathrm{x}_{\mathrm{Fe}}{ }^{0} \mathrm{G}_{\mathrm{Fe}}^{\phi}+\mathrm{x}_{\mathrm{Mn}}{ }^{0} \mathrm{G}_{\mathrm{Mn}}^{\phi}+\mathrm{RT}\left(\mathrm{x}_{\mathrm{Co}} \ln \mathrm{x}_{\mathrm{Co}}+\mathrm{x}_{\mathrm{Fe}} \ln \mathrm{x}_{\mathrm{Fe}}+\mathrm{x}_{\mathrm{Mn}} \ln \mathrm{x}_{\mathrm{Mn}}\right) \\
& +\mathrm{x}_{\mathrm{Co}} \mathrm{x}_{\mathrm{Fe}} \mathrm{L}_{\mathrm{Co}, \mathrm{Fe}}^{\phi}+\mathrm{x}_{\mathrm{Co}} \mathrm{x}_{\mathrm{Mn}} \mathrm{L}_{\mathrm{Co}, \mathrm{Mn}}^{\phi}+\mathrm{x}_{\mathrm{Fe}} \mathrm{x}_{\mathrm{Mn}} \mathrm{L}_{\mathrm{Fe}, \mathrm{Mn}}^{\phi}+\Delta \mathrm{G}_{\mathrm{m}}^{\mathrm{mg}, \phi}
\end{aligned}
$$

where $\mathbf{x}_{\mathbf{i}}(\mathrm{i}=\mathrm{Fe}, \mathrm{Mn}, \mathrm{Co})$ is the atomic fraction of the element $\mathrm{i}$ and ${ }^{0} \mathrm{G}_{i}^{\phi}$ is the Gibbs energy of the element $\mathrm{i}$ with the structure of the phase $\phi$ in a non-magnetic state and $\Delta G^{m g}$ is the magnetic contribution. The information about ${ }^{0} \mathrm{G}_{\mathrm{i}}^{\phi}$ was taken from assessments of the thermodynamic properties of $\mathrm{Fe}$ [10], Co [11] and $\mathrm{Mn}$ [12]. The magnetic contribution $\Delta \mathrm{G}_{\mathrm{m}}^{\mathrm{mg}, \phi}$ was described by using the Hillert-Jarl [13] phenomenological model, which gives

$$
\Delta \mathrm{G}_{\mathrm{m}}^{\mathrm{mg}, \phi}=\mathrm{RT} \ln \left(\beta^{\phi}+1\right) \mathrm{f}^{\phi}\left(\tau^{\phi}\right)
$$

where $\beta^{\phi}$ is a composition dependent parameter related to the total magnetic entropy, i.e., the quantity $\Delta \mathrm{S}_{\mathrm{m}}^{\mathrm{mg}, \phi}(\infty)-\Delta \mathrm{S}_{\mathrm{m}}^{\mathrm{mg}, \phi}(0)$, as follows

$$
\Delta S_{\mathrm{m}}^{\mathrm{mg}, \phi}(\infty)-\Delta \mathrm{S}_{\mathrm{m}}^{\mathrm{mg}, \phi}(0)=\mathrm{R} \ln \left(\beta^{\phi}+1\right)
$$

The variable $\tau^{\phi}$ is defined as $\mathrm{T} / \mathrm{T}_{\mathrm{N}}^{\phi}$ where $\mathrm{T}_{\mathrm{N}}^{\phi}$ is the Néel temperature of $\phi$ (fcc or hcp). When applying the model to alloys, $\beta^{\phi}$ and $\mathrm{T}_{\mathrm{N}}^{\phi}$ are to be expressed as functions of composition. This was done as explained in refs.[14] and [15]. The composition dependent $L_{i j}^{\phi}(i, j=C o, F e, M n)$ parameters in Eq. (1) originate in the various binary subsystems of the present ternary, and account phenomenologically for the deviations from the ideal behaviour in the non-magnetic Gibbs energy. The $L_{\mathrm{ij}}^{\phi}$ parameters for the fcc phase of the Fe-Co and $\mathrm{Mn}-\mathrm{Co}$ systems were directly taken from refs.[14] and [16], respectively. The fcc phase of the Fe-Mn system was treated using the $\mathrm{L}_{\mathrm{Fe}, \mathrm{Mn}}^{\mathrm{fcc}}$ value from ref. [17], but its $\beta^{\text {fcc }}$ vs. composition function was taken from our previous work $[6,7]$. The hcp phase in the Fe-Mn and Mn-Co systems was described using the 
$\mathrm{L}_{\mathrm{ij}}^{\mathrm{hcp}}$ parameters reported in refs. [6, 7] and [16], respectively. For the description of the binary hcp phase of the Fe-Co system a subregular solution approximation (SSA), given by,

$$
\mathrm{L}_{\mathrm{Co}, \mathrm{Fe}}^{\mathrm{hcp}}={ }^{0} \mathrm{~L}_{\mathrm{Co}, \mathrm{Fe}}^{\mathrm{hcp}}+{ }^{1} \mathrm{~L}_{\mathrm{Co}, \mathrm{Fe}}^{\mathrm{hcp}}\left(\mathrm{x}_{\mathrm{Co}}-\mathrm{x}_{\mathrm{Fe}}\right)
$$

was adopted. In Eq. (4) ${ }^{0} \mathrm{~L}_{\mathrm{Co}, \mathrm{Fe}}^{\mathrm{hcp}}$ and ${ }^{1} \mathrm{~L}_{\mathrm{Co}, \mathrm{Fe}}^{\mathrm{hcp}}$ are composition independent parameters that account phenomenologically for the interaction between $\mathrm{Fe}$ atoms and $\mathrm{Co}$ atoms. These parameters were treated as independent of temperature and determined from experimental data, as described in the following subsection.

\subsection{Parameter Evaluation Procedure}

The evaluation of the ${ }^{0} \mathrm{~L}_{\mathrm{Co}, \mathrm{Fe}}^{\mathrm{hcp}}$ and ${ }^{1} \mathrm{~L}_{\mathrm{Co}, \mathrm{Fe}}^{\mathrm{hcp}}$ parameters was performed by a computer optimisation procedure. The program [18] minimised the sum of the squares of the differences between experimental and calculated values of the $T_{0}$ temperature. The experimental $T_{0}$ 's were estimated as $\left(M_{s}+A_{s}\right) / 2$, and the calculated ones were obtained by solving the equation:

$$
\mathrm{G}_{\mathrm{m}}^{\mathrm{hcp}}\left(\mathrm{T}_{0}, \mathrm{x}_{\mathrm{Mn}}, \mathrm{x}_{\mathrm{Co}_{0}}\right)=\mathrm{G}_{\mathrm{m}}^{\mathrm{lic}}\left(\mathrm{T}_{0}, \mathrm{x}_{\mathrm{Mn}}, \mathrm{x}_{\mathrm{CO}_{0}}\right)
$$

which defines the $\mathrm{T}_{0}$ temperature for the fcc/hcp transformation. The database used in the optimisation amounted to eleven values, among which seven values correspond to low-Mn Fe-Mn-Co alloys and four values to $\mathrm{Fe}-\mathrm{Co}$ alloys. The parameters that minimised the error sum are as follows:

$$
{ }^{0} \mathrm{~L}_{\mathrm{Co}, \mathrm{Fe}}^{\mathrm{hcp}}=-7733.6 \mathrm{~J} / \mathrm{K} \cdot \mathrm{mol} \quad{ }^{1} \mathrm{~L}_{\mathrm{Co}, \mathrm{Fe}}^{\mathrm{hcp}}=1105.4 \mathrm{~J} / \mathrm{K} \cdot \mathrm{mol}
$$

\section{DISCUSSION}

\subsection{The Fe-Mn-Co System}

The $\mathrm{T}_{0}$-lines for Fe-Mn-Co alloys, calculated by solving Eq. (5) with the optimum Gibbs energy functions are compared in Fig. 2 with experimental data. For alloys with $\mathrm{Mn}$ contents such that $17 \leq \mathrm{wt} . \% \mathrm{Mn} \leq 25$, Fig. 2 shows a general agreement between the calculated $\mathrm{T}_{0}$ and the values obtained from experiments as $\left(A_{s}+M_{s}\right) / 2$. In particular, the temperatures corresponding to alloys represented by triangles are well accounted for by the calculation, which is natural because these values were included in the optimisation procedure. However, there are other points that are placed at high and at low Co contents

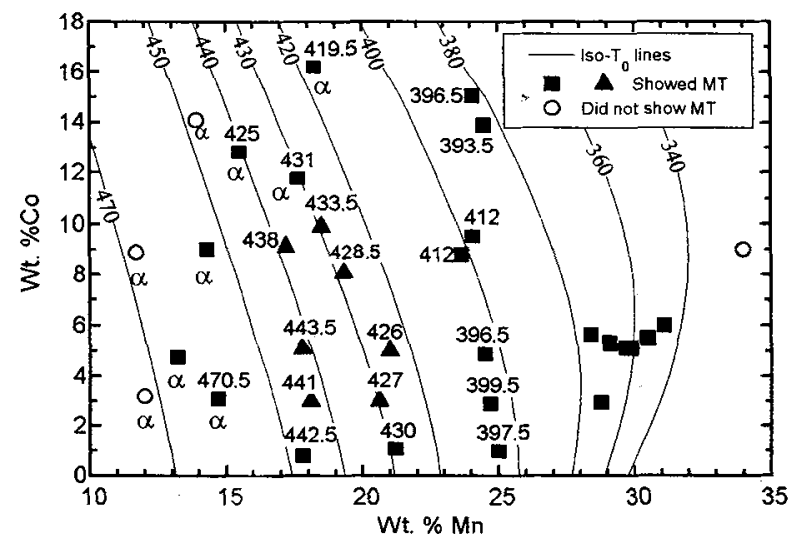

Fig. 2: Calculated iso- $T_{0}$ lines and $T_{0}$ values estimated from experiments as (filled symbols). The triangles represent the alloys used for the calculat ons. The empty circles represent the alloys that do not show the fcc/licp martensitic transformation. in the given $\mathrm{Mn}$ range, which were not included in the optimisation, but fall close to the calculated lines. Such an agreement suggests that: (i) the magnetic effects, which are expected [6] to cause differences between $T_{0}$ (Eq. (6)) and $\left(A_{s}+M_{s}\right) / 2$, are not strong in these alloys, and (ii) the formation of the $\alpha$ martensite has not significantly affected the $M_{s}$ and $\mathrm{A}_{\mathrm{s}}$ temperatures for the fcc/hcp MT. The discrepancies between experiments and calculations that appear to the left of Fig. 2 are probably due to the presence of $\alpha$ martensite, whereas the magnetic effect is expected to be 
the main cause of differences for the alloys appearing to the right of Fig.2. In spite of the expected discrepancies with the $\left(A_{s}+M_{s}\right) / 2$ approximation, the theoretical $T_{0}$ values obtained from Eq. (6), satisfy the condition $M_{s}<T_{0}<A_{s}$ even in the presence of strong magnetic effects. This is illustrated, in particular, by the group of alloys with $380 \mathrm{~K}>\mathrm{T}_{0}>340 \mathrm{~K}$. Finally, the empty circles in Fig. 2 indicate those alloys that did not show fcc/hcp MT. In the case of the two alloys located at the left of Fig.2, the occurrence of fcc/ $\alpha$ MT was observed, which has probably hampered the fcc/hcp MT. The alloy with the highest Mn content among those studied by us has a theoretical $\mathrm{T}_{0}$ value of $318 \mathrm{~K}$. However the fcc/hcp MT was not observed in spite of the fact that the samples were cooled down by immersion in liquid nitrogen. Two possible explanations of the discrepancy can be offered: (i) the $\mathrm{M}_{\mathrm{s}}$ temperature for this alloy is lower than $78 \mathrm{~K}$, i.e., the lowest temperature achieved on cooling, and (ii) the amount of hcp phase formed is so low that it could not be detected in our electrical resistivity or dilatometric measurements. New experiments in the high-Mn range are under way which should help to decide among these explanations.

\subsection{The Fe-Co System}

In a previous work [10], one of us (AFG) assessed the Gibbs energy function of the hcp phase in the FeCo system using the regular solution approximation (RSA), and determining the interaction parameter by fitting to the only piece of experimental information then available on this phase, namely, the $\mathrm{T}_{0}$ temperatures that can be estimated from fcc/hcp MT data. More recently it was found by us [5] that such [10] description of the hcp phase leads to ternary $T_{0}$ temperatures that are not compatible with the MT data from Fe-Mn-Co alloys [5]. As a consequence, an attempt was made by us [5] to describe consistently the binary and the ternary

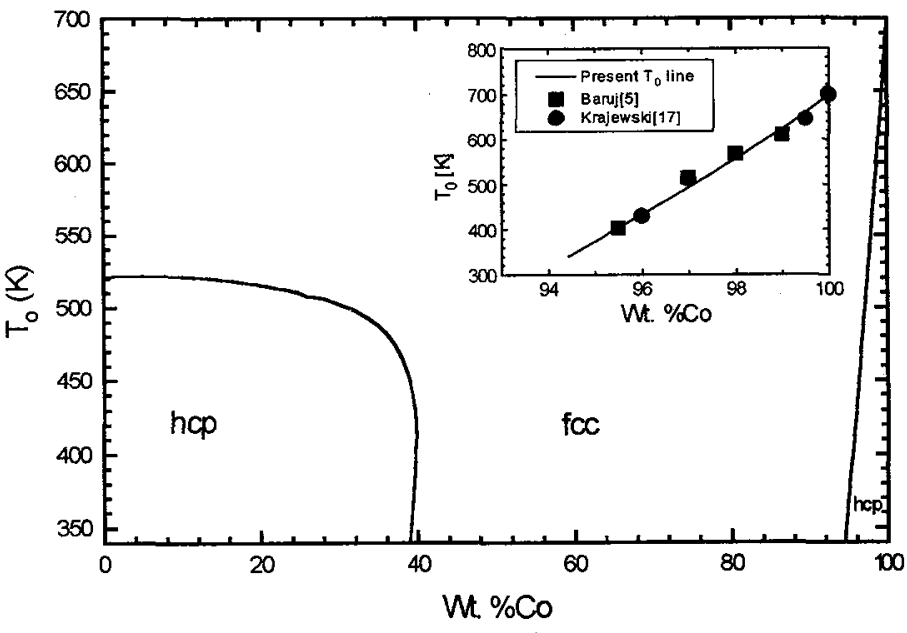

Fig. 3: The $\mathrm{T}_{0}$ line resulting from present calculation for the Co-Fe system. As an inset the comparison with experimental $T_{0}$ taken from refs. $[5,16]$ is given. information, by describing the binary hep phase in terms of the subregular solution approximation (SSA). These ideas are now applied to a considerably expanded database, which contains new ternary data as well as the recently presented $\mathrm{M}_{\mathrm{s}}$ and $\mathrm{A}_{\mathrm{s}}$ temperatures for the fcc/hcp $\mathrm{MT}$ in Co-rich Fe-Co alloys [5]. The $\mathrm{T}_{0}$ versus composition relations calculated by using the optimum interaction parameters (Eq.(6)) for the FeCo system are plotted in Fig. 3. The inset there gives a comparison between the calculated $\mathbf{T}_{0}$-line and the values estimated from experiments $[5,19]$, some of which were included in the optimisation procedure. The calculation accounts well even for the data not used in determining ${ }^{0} \mathrm{~L}_{\mathrm{Co}, \mathrm{Fe}}$ and ${ }^{1} \mathrm{~L}_{\mathrm{Co}, \mathrm{Fe}}^{\mathrm{hcp}}$. The $\mathrm{T}_{0}$-line at the Co-rich side of the system shows a rapid decrease with increasing Fe content, but a smaller rate of change with the initial additions of $\mathrm{Co}$ is predicted for the Fe-rich alloys. Such an asymmetry can be rationalized by considering the magnetic contributions to phase stability in the Fe-Co system. The Fe-rich fcc phase is antiferromagnetic with a very low $\mathrm{T}_{\mathrm{N}}$, but becomes ferromagnetic at about 15 at $\%$ Co [10]. The corresponding Curie temperature $\left(\mathrm{T}_{\mathrm{c}}\right)$ and magnetic entropy increases with further Co additions, and becomes equal to the presently calculated $\mathrm{T}_{0}$ at about $40 \mathrm{wt} \% \mathrm{Co}$, i.e., the composition at which the dramatic stabilization of fcc sets in. For Co contents larger than about $40 \mathrm{wt} \%$, the ferromagnetic fcc phase is more stable than hcp, except for a very narrow range close to pure Co. There, an hcp phase which is 
ferromagnetically ordered, and shows magnetic contributions to Gibbs energy comparable to those of the competing fcc structure [11], becomes the stable phase.

\section{SUMMARY AND CONCLUDING REMARKS}

Traditionally, thermodynamic information on materials has been extracted from direct thermochemical measurements, and from experimental data on the phase diagram. However, when assessing the thermodynamics of a phase one aims at establishing the Gibbs energy function even in regions of the space of variables in which such a phase is not the most stable one. This might be achieved if some indirect information about the behaviour in the regions of non-stability is obtained and included in the assessment. In the present work, new information has been obtained about the stability of a prototype phase, viz., the hcp structure in the Fe-Mn-Co system. Hcp is the stable phase only at the Co-rich corner of the ternary phase diagram, but has now been studied in a wide range of compositions, which includes Fe-Mn-Co alloys with very low Co contents. The approach combines two main objectives: (i) forming the non-stable hep structure from the stable fcc phase through a MT, and (ii) extracting thermodynamic information from the MT temperatures. Indeed, these two aspects have previously been dealt with in other systems. However, a specific goal of the present work was to deal with the experimental and theoretical complications caused by the existence of strong magnetic effects. Formally, these effects have been accounted for by adopting a phenomenological description of the magnetic contribution to Gibbs energy. Furthermore, the non-magnetic part of the Gibbs energy has been treated with a substitutional solution model which includes only binary contributions, but does not invoke ternary interactions. This assumption, which has proved to be consistent with the available data on the stable phases of the Fe-Mn-Co system, has now been applied in the treatment of the hcp structure in the regions where it is not a stable phase. In this way it has been possible to obtain a refined picture of the metastable phase relations in the Fe-Co system.

\section{References}

[1] Hamers A. A. H. and Wayman C. M., Scripta Met. and. Mater., 25 (1991), pp. 2723-2728.

[2] Chen C. F., Yang J. H. and Zhao L., MRS., 9 (1988), pp. 481-48

[3] Ishida K. and Nishizawa T., Trans. JIM, 15 (1974), pp. 225-31.

[4] Fernández Guillermet A., High Temp.- High Press., 19 (1987), pp. 477-499.

[5] Baruj A., Cotes S., Sade M. and Fernández Guillermet A., Journal de Physique IV, Colloque C8, 5 (1995), pp. 373-378.

[6] Cotes S. M., Baruj A., Sade M. and Fernández Guillermet A., Journal de Physique IV, Colloque C2, 5 (1995), pp. 83-88.

[7] Cotes S. M., Sade M. and Fernández Guillermet A., Metallurgical and Materials Transactions A, 26A (1995), pp. 1957-1969.

[8] Baruj A., Cotes S., Sade M. and Fernández Guillermet A., Z. Metallkd., 87 (1996) 10, pp. 765-772

[9] Khomenko, Khil'kevich I.F. and Zvigintseva G. Ye., Fiz. Metal. Metalloved., 37, No.6 (1974), p.1325.

[10] Fernández Guillermet A., Gustafson P. High Temp.-High-Press., 6 (1985), pp. 591-610.

[11] Fernández Guillermet A., Internat. Journ. Thermophysics, 8 (1987), pp. 481-510.

[12] Fernández Guillermet A. and Huang W., Internat. Journ. Thermophysics, 11 (1990), pp. 949-69.

[13] Hillert M. and Jarl M., Calphad, 2 (1978), pp. 227-238.

[14] Fernández Guillermet A., High Temp.- High Press., 19 (1987), pp. 477-499.

[15] Huang W., CALPHAD, 14 (1990) 1, pp. 11-22.

[16] Huang W., CALPHAD, 13 (1989), pp. 231-242.

[17] Huang W., CALPHAD, 13 (1989), pp. 243-252.

[18] Jansson B., Ph. D. Thesis, The Royal Institute of Technology, Stockholm, Sweden (1984).

[19] Krajewski W., Krüger J. and Winterhager H., Metall. J., 24 (1970), pp. 480-487. 